\title{
Long-Term Latency Measurement of Deployed Fiber
}

\author{
Florian Azendorf ${ }^{1}$, Annika Dochhan ${ }^{1}$, Ralf-Peter Braun ${ }^{2}$, Michael Eiselt ${ }^{1}$ \\ ${ }^{1}$ ADVA Optical Networking SE, Maerzenquelle 1-3, 98617 Meiningen, Germany \\ ${ }^{2}$ Deutsche Telekom AG, Winterfeldtstraße 21, 10781 Berlin, Germany \\ fazendorf@advaoptical.com
}

\begin{abstract}
Using a Correlation-OTDR we measured the latency of fibers in a deployed cable and calculated the time coefficient of the fiber temperature changes. Annual temperature variations of $25 \mathrm{~K}$ were estimated for the deployed fiber.

OCIS codes: (060.2270) Fiber Characterization; (120.4825) Optical Time Domain Reflectometry.
\end{abstract}

\section{Introduction}

Latency is becoming a critical parameter in future $5 \mathrm{G}$ networks. Synchronization protocols require a stable and symmetric latency between master and slave clocks. Other applications, like the transmission of radio phase array signals, as investigated in the European BlueSpace project [1], require a very low differential latency between different optical paths. One of the factors strongly impacting latency is temperature, which mainly impacts the refractive fiber index, resulting in a latency change of approximately $6 \mathrm{ppm} / \mathrm{K}[2,3]$. In addition, strain effects in a jumper cable with a tight buffer can lead to a temperature delay coefficient (TDC) of $17 \mathrm{ppm} / \mathrm{K}$, as measured in a laboratory environment [4]. So far, to our knowledge, the latency changes of a fiber deployed in the ground has not been measured with a high accuracy. In this paper, we report on those measurements, using a Correlation-OTDR (C-OTDR), yielding a high accuracy of the absolute fiber latency on the order of a few picoseconds [5].

\section{Experimental setup}

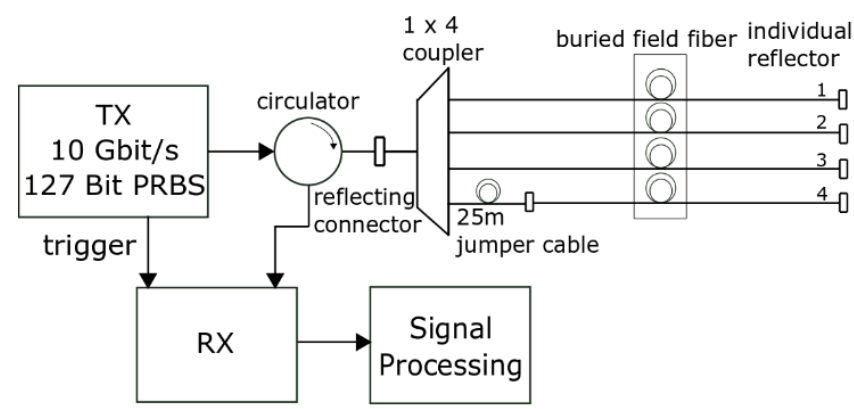

Fig. 1: Measurement setup for simultaneous latency characterization of four deployed fibers.

We characterized four fibers in a deployed $8.5-\mathrm{km}$ underground cable between the ADVA office and a Deutsche Telekom central office in Meiningen, in Central Germany. The setup is shown in Fig. 1. A laser at a wavelength of $1550 \mathrm{~nm}$ was modulated using a Mach-Zehnder modulator with a 10-Gbit/s 127-bit PRBS burst followed by zeros to fill a $100-\mu$ s packet, sufficient to measure a $10-\mathrm{km}$ fiber. The signal was sent via a circulator and passed a connector with an air gap, providing a reference reflection from the near end of the fiber. To measure all fibers simultaneously, the signal was split using a 1x4-coupler. Fiber loops using 1x2-couplers provided a high reflection from the far end of each fiber (individual reflectors). The reflected light was received after the circulator and, after a PIN/TIA receiver, observed on a 50-GS/s real time oscilloscope. The real time oscilloscope was synchronized with the burst trigger from the PRBS source, and 2000 traces were recorded and averaged. The averaged signal is shown in Fig. 2a, showing the PRBS sequences reflected from the four fiber ends. Note that the first two reflected packets are overlapping. The averaged signal was correlated with the transmitted PRBS and filtered to eliminate pre- and post-cursors in the correlation function due to the isolated PRBS. The correlated signal is shown in Fig. 2b. As the lengths of two fibers matched within less than 100 ps such that their end reflections were indistinguishable, we added a 25-m jumper cable to the $4^{\text {th }}$ fiber. The resulting higher round-trip latency of $250 \mathrm{~ns}$ can be seen in Fig. $2 \mathrm{~b}$. As the 50 GS/s oscilloscope 
yielded only a sample resolution of $20 \mathrm{ps}$, a raised cosine function was fitted to the highest correlation peaks to improve the position accuracy to a few picoseconds [5]. The cosine fit is show in Fig. 2c.
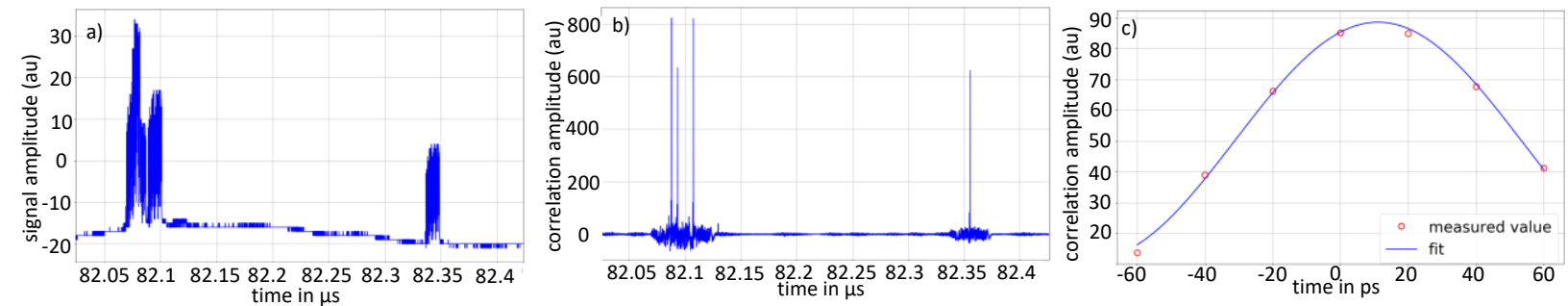

Fig. 2: a) Averaged signal showing reflected PRBS sequences; b) averaged signal correlated with transmitted PRBS sequence showing reflection peaks from four fiber ends; c) fit of one correlation peak to a raised cosine function.

\section{Results}

Using the C-OTDR, the round-trip latency of the four fibers was monitored over approximately two weeks with a half day interrupted around the $8^{\text {th }}$ day. As shown in the blue curve in Fig. 3a, the maximum latency increase over the time, referenced to the latency at the start of the measurement, was 800 ps. Fig. 3a also contains the outside air temperature, as obtained from a data base maintained by the German Meteorological Service [7]. It can be seen that the latency does not immediately follow the temperature but is dampened and has some time delay. Similar behavior was reported in [6], where the temperature in soil in different depths was measured. We modelled the temperature variation of the fiber as a low-pass filtered response to the outside temperature, as reported in Section 4 of this paper. This delayed response of the fiber temperature led to daily latency variations of about $200 \mathrm{ps}$. All fibers exhibit similar behavior, as shown in detail in Fig. $3 \mathrm{~b}$ for a 12 -hour period. However, fiber 4 shows a larger latency variation, which we attribute to the additional $25 \mathrm{~m}$ of jumper cable, which was subjected to the laboratory environment, resulting in a round-trip latency variation of approximately $10 \mathrm{ps} / \mathrm{K}$ [2]. The latency difference of $20 \mathrm{ps}$ corresponds to a temperature increase of $2 \mathrm{~K}$, which is a typical value in our laboratory over the day.
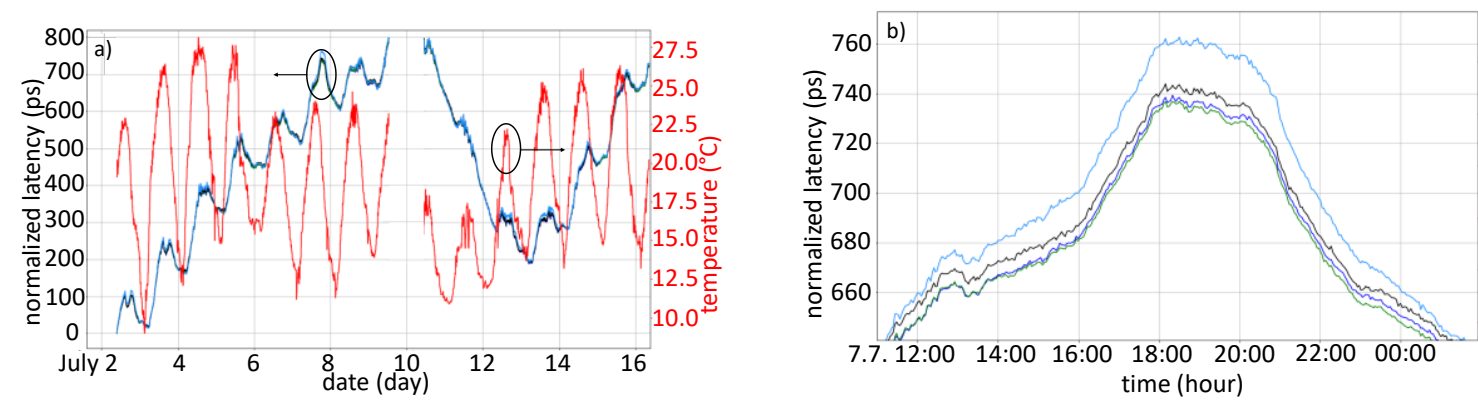

Fig. 3: Results of the long-term measurements; a.) relative round-trip latency of four deployed fibers and outside temperature over two weeks in July 2018 with a half day interruption from $9^{\text {th }}$ to $10^{\text {th }} ; \mathrm{b}$.) latency evolution of four fibers on July $7^{\text {th }}$.

Another important parameter for synchronization applications is the latency difference (or skew) between the fibers. Fig. 4 shows the skew variations between fibers 1,2, and 3 over the measurement period, offset by the minimum skew. The maximum variation was approximately $12 \mathrm{ps,} \mathrm{seen} \mathrm{between} \mathrm{fibers} 1$ and 3 . It can also be seen that the skew

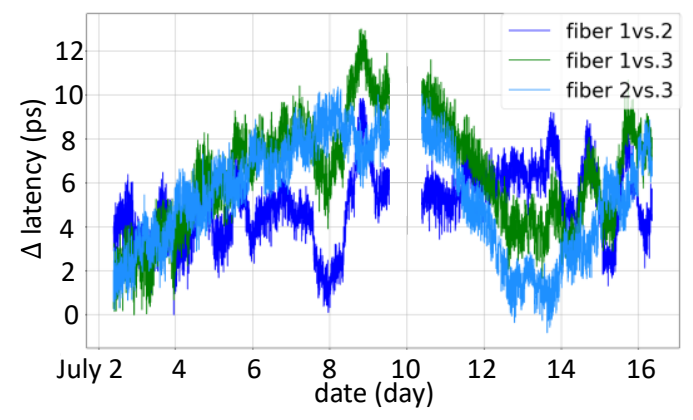

Fig. 4: Variation over time of differential latency (skew) between fibers 1, 2, and 3. 
variation approximately tracks the absolute latency changes (blue curve in Fig. 3a) and might be attributed to TDC differences between the fibers on the order of $1 \%$.

\section{Modelling of the fiber temperature}

We modelled the evolution of the temperature of the fiber deployed in the ground as a $1^{\text {st }}$ order low-pass filtered function of the outside air temperature. The low-pass filter parameters were derived by a fit of the filtered temperature to the measured latency variations of the fiber. This assumes that the latency variations are linear with the fiber temperature, which was established e.g. in [4]. Fig. 5a shows the measured latency variation as well as the filtered temperature changes with fitting parameters of $7.5 \mathrm{ppm} / \mathrm{K}$ for the TDC and 12.7 days for the filter time constant. It can be seen that the modelling replicates the slower trends of latency changes over multiple days as well as some of the smaller day-night variations. The peak-to-peak temperature variation of the cabled fiber was only $1.5 \mathrm{~K}$ during the two weeks of measurement. Using this model for the annual variations of the fiber temperature based on data of the air temperature obtained from the German Meteorological Service [7], we estimated the variation of the fiber temperature over the year. Fig. $5 \mathrm{~b}$ shows the air temperature variation with a resolution of 10 minutes and the resulting filtered fiber temperature with a peak-to-peak variation of approximately $28 \mathrm{~K}$. This temperature variation would lead to a round-trip latency variation of the $8.5-\mathrm{km}$ fiber link by approximately $17 \mathrm{~ns}$ over the year. A skew variation of approximately 200 ps can be estimated based on the results in Figure 4.
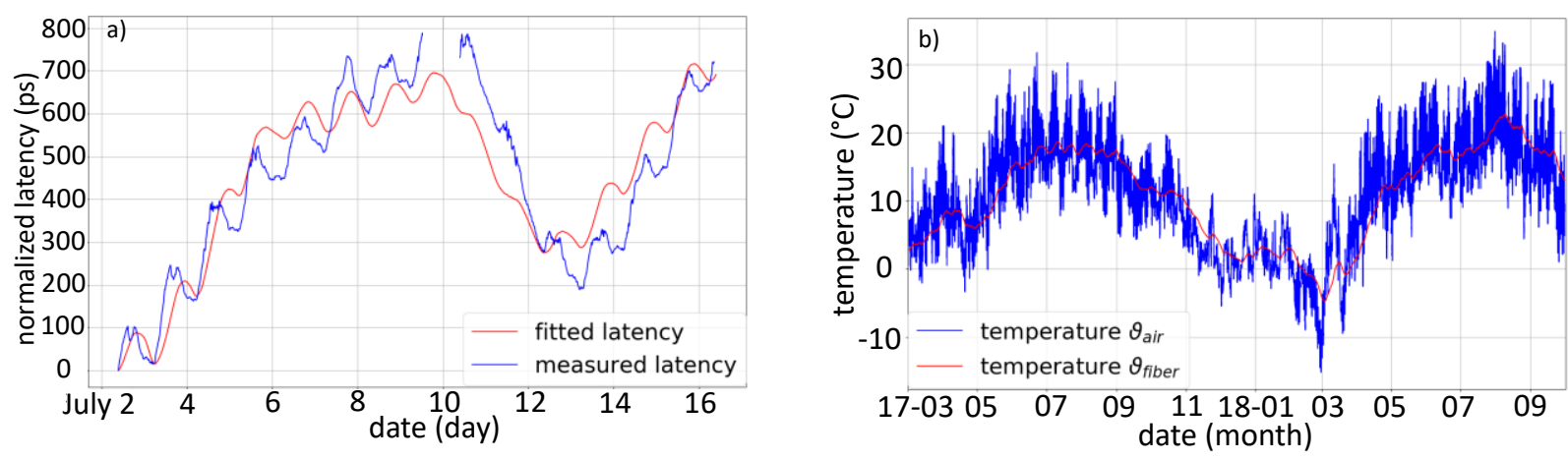

Fig. 5: Modelling of cabled fiber temperature a) Measured fiber latency(blue) and latency based on filtered cable temperature (red); b) Temperature evolution over 18 months: 10-min air temperature data(blue) and slower changing fiber temperature (red).

\section{Summary}

Using a correlation OTDR, with an accuracy of approximately $2 \mathrm{ps,} \mathrm{we} \mathrm{measured} \mathrm{the} \mathrm{latency} \mathrm{variations} \mathrm{of} \mathrm{four} \mathrm{fibers}$ in a deployed $8.5-\mathrm{km}$ cable. Over a period of 14 days in the summer, a maximum round-trip latency variation of $800 \mathrm{ps}$ was measured. The measured skew variations between the fibers were up to $12 \mathrm{ps}$. Based on these measurements, a time constant of 12.7 days for the temperature change of the fibers in the cables was extracted, which would lead to a temperature change of $28 \mathrm{~K}$ over the year, resulting in variations of latency and skew of $17 \mathrm{~ns}$ and $200 \mathrm{ps}$, respectively.

\section{Acknowledgment}

This project has received funding from the European Union's Horizon 2020 research and innovation programme under grant agreement No 762055 (BlueSpace Project).

\section{References}

[1] https://bluespace-5gppp.squarespace.com/

[2] J. W. Dreher, "Phase Stability of ATA Fiber Optic Cables", SETI Institute, November 2000

[3] G. Lutes and W. Diener, "Thermal coefficient of delay for various coaxial and fiber-optic cables," TDA Progress Report 42-99, Nov. 1989, available online at https://ipnpr.jpl.nasa.gov/progress_report/42-99/99E.PDF

[4] F. Azendorf, A. Dochhan, and M. Eiselt, “Temperature Dependent Latency of Jumper Cable “, ITG Photonic Networks 2018, Leipzig, June 2018.

[5] M. Eiselt and A. Dochhan, „Single-Ended Fiber Latency Measurement with Picosecond-Accuracy Using Correlation OTDR,” paper 5C1-3, Proc. 23st OptoElectronics and Communications Conference (OECC), Jeju, Korea, July 2018.

[6] G. Florides and S. Kalogirou, "Annual Ground Temperature Measurement at Various Depths", $8^{\text {th }}$ REHVA World Congr. 2005, available on https://www.researchgate.net/publication/30500353

[7] ftp://ftp-cdc.dwd.de/pub/CDC/observations germany/climate/10 minutes/air temperature/recent/ 